\title{
Les apports des techniques structurales à l'analyse des processus d'innovation : une introduction
}

\section{The contributions of structural techniques to the analysis of innovation}

\author{
processes: an introduction
}

\author{
Didier Lebert ${ }^{1}$ \\ ${ }^{1}$ Unité d'Economie Appliquée, ENSTA Paris, i3-CRG Ecole Polytechnique - CNRS (UMR 9217), Institut Polytechnique de \\ Paris, France, didier.lebert@ensta-paris.fr
}

\begin{abstract}
RÉSUMÉ. Ce numéro de Techlnn est dédié aux outils de l'analyse structurale appliqués au domaine de l'économie de l'innovation. II comprend six articles. Le message essentiel qu'ils portent est que ces outils ne servent pas seulement à dessiner / visualiser des relations entre les nœuds d'un réseau. Elles servent également d'aide à la prise de décision pour les industriels et les pouvoirs publics en matière de stratégie et de politique d'innovation.

ABSTRACT. This issue of Techlnn is dedicated to the tools of structural analysis applied to the economics of innovation. It includes six articles. The essential message they carry is that these tools are not only used to draw / visualize relationships between nodes in a network. They also serve as a decision-making aid for companies and public authorities in terms of innovation strategy and policy.

MOTS-CLÉS. Analyse des réseaux sociaux, théorie de la dominance économique, brevets.

KEYWORDS. social network analysis, economic dominance theory, patents.
\end{abstract}

[KRA 11] représentent la structure et la dynamique d'un système sectoriel d'innovation - celui des biotechnologies - en utilisant les techniques de l'analyse des réseaux sociaux [WAS 94]. [ELO 16] mobilisent cette même représentation pour comprendre la dynamique de spécialisation intelligente des régions mise en œuvre dans le cadre de la politique d'innovation européenne. [FRI 19] font de même pour étudier la formation d'un système technologique d'innovation - le véhicule à pile à combustible à l'échelle internationale sur la base des co-dépôts de brevets. Dans tous ces exemples, une structure est dessinée sous la forme d'un réseau ou d'un « graphe » : des entités / nœuds (représentant ici des technologies, des entreprises ou des régions) sont reliées entre elles par des liens.

Deux grandes manières d'appréhender ces liens entre nœuds sont privilégiées dans la littérature. Ils représentent quelquefois des flux au sein du réseau. Ces flux (physiques, monétaires, de connaissances) sont représentés sous la forme de liens dirigés de l'entité émettrice vers l'entité réceptrice et pondérés par leur intensité. D'autres fois, ces liens représentent des cooccurrences de caractéristiques (la présence des mêmes combinaisons technologiques dans un portefeuille de brevets par exemple, comme dans l'exemple de [KRA 11] ci-dessous). Ces cooccurrences sont représentées sous la forme de liens non dirigés et pondérés par leur nombre.

A ces représentations dites structurales sont associés de nombreux indicateurs dédiés. Les termes de « degré », « force », « densité », « proximité » (closeness), « connexité », « assortiment / homophilie », « centralité », « intermédiarité », et de nombreux autres propres à la sociométrie, ont permis de porter un regard neuf sur les enjeux associés à l'organisation et aux dynamiques territoriales et industrielles, aux pratiques et à l'organisation des activités d'innovation, à leur diffusion, leur qualité, et aux stratégies et politiques qui les soutiennent. 


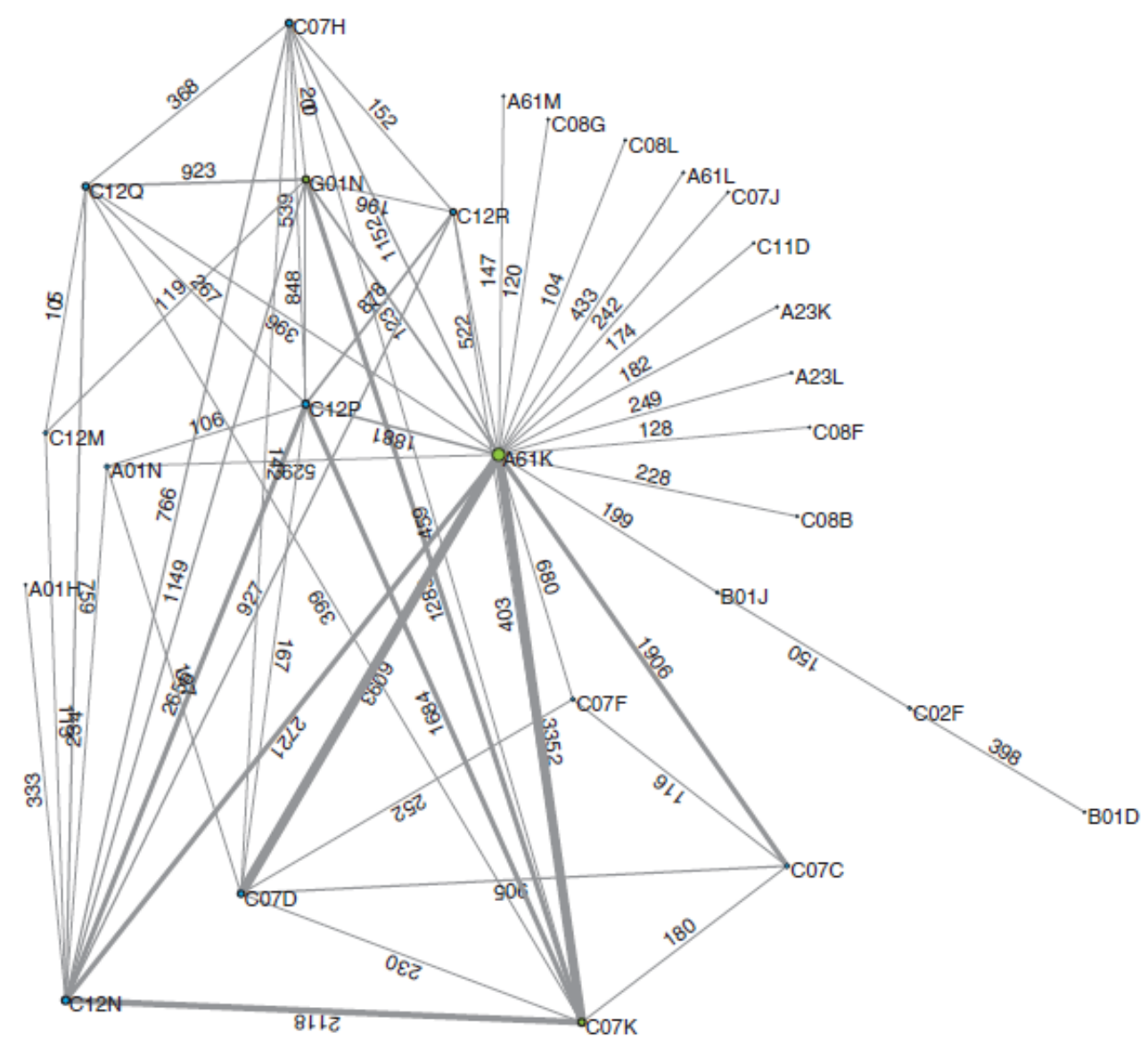

Figure 1. Exemple d'un réseau de cooccurrences dans le secteur des biotechnologies [KRA 11]

Lecture. Le graphe ou réseau est construit à partir de données de brevets. Les nœuds du graphe sont des technologies (nomenclature CIB à 4 digits), les liens dénotent l'existence d'inventions combinant ces technologies, et l'épaisseur de ces liens indique l'intensité de ces combinaisons, information sur l'intensité qui est également révélée par le nombre au milieu de chaque lien.

Ce sont ces représentations et outils d'analyse appliqués au domaine de l'économie de l'innovation qui sont au cœur de ce numéro de Technologie et Innovation. Le numéro s'intitule « Les apports des techniques structurales à l'analyse des processus d'innovation » et il comprend six articles. Le message essentiel que portent les contributions à ce numéro, selon moi, est que les techniques structurales ne servent pas seulement à dessiner / visualiser des relations entre les nœuds d'un réseau. Elles servent également de base à l'élaboration d'outils d'aide à la prise de décision pour les industriels et les pouvoirs publics en matière de stratégie et de politique d'innovation.

Frédéric Caillaud et Clément Sternberger ( $«$ La carte d'État-major des inventions brevetées : un avantage concurrentiel majeur pour innover ») recensent les usages des cartographies de brevets que proposent certains logiciels manipulant de la donnée brevet brute pour la rendre intelligible afin de définir ou d'évaluer une stratégie d'innovation pour l'entreprise (Questel ORBIT, Clarivate Derwent, etc.). A première vue, les techniques structurales sont absentes de ces solutions. Dans les faits, elles sont bien en leurs cœurs, mais elles restent invisibles aux yeux des usagers. Les techniques structurales contribuent en effet à établir des distances entre brevets en fonction de leurs contenus sémantiques. Cela s'apparente à de la cooccurrence. En s'appuyant sur une longue pratique de ces cartographies dans des domaines d'expertise ciblés, les auteurs montrent les potentialités ainsi que les pièges que peut receler la lecture de ces cartes. La production des cartes et des différents indicateurs permettant d'en faciliter la lecture ne doit surtout pas se substituer au regard de l'expert technologique et sectoriel, à même de les mettre en perspective. 
C'est à une revue de littérature large sur les graphes de cooccurrences dans leurs usages les plus récents en économie de l'innovation que se livre Cécile Fauconnet (« Les graphes de cooccurrences pour l'analyse de l'innovation »). Elle identifie des champs d'application qui ont pour particularité de s'être développés au cours des dix dernières années en lien avec ces techniques structurales : l'organisation d'un domaine technologique, la "cohérence » de la R\&D d'un grand groupe, la cartographie «technologique » des inventions, et la diffusion technologique. Ce qui me semble être au cœur de l'usage de ces méthodes est la compréhension de la dynamique des structures : comment un domaine technologique, ou la cohérence technologique d'une entreprise par exemple, se transforment ? Les graphes de cooccurrences proposent des quantifications de ces transformations, l'étape suivante consistant à les insérer dans des modèles explicatifs multidimensionnels.

Raffaele Anedda s'attaque à un problème ardu : la mesure de la qualité d'une invention («La qualité technologique des brevets : deux lectures structurales »). Ce point a donné lieu à des publications STI de l'OCDE durant la dernière décennie. Les discussions continuent, non encore définitivement tranchées. Ce qui est sûr, c'est que l'outil « graphe » est absent des développements sur cette question. Raffaele veut montrer que cette absence n'est que relative. On retrouve en effet du réseau dans la mesure des indicateurs classiques de la qualité. Le réseau en question est un réseau de flux particulier : à un brevet donné s'associe un ensemble de technologies plus anciennes qui ont été mobilisées pour le produire (" originalité » d'une invention) ou bien qui sont produites en le mobilisant (" généralité » d'une invention). Ce réseau de flux de citations est particulier car l'identité des technologies allouées au brevet évalué n'entre pas en considération. Que se passerait-il si on la prenait en compte ? Raffaele montre qu'on enrichirait substantiellement la portée des indices d'originalité et de généralité, et on pourrait concevoir de nouveaux indices reposant entièrement, cette fois-ci, sur une approche structurale. Il en propose quelques-uns lui-même, qui ne demandent plus qu'à être appliqués sur des bases de données étendues ... et soumis au regard de l'expert, cf. supra.

Je contribue à ce numéro en présentant une technique nouvelle pour identifier des arbres aux propriétés remarquables au sein des réseaux de flux ( «Les arbres couvrants d'un graphe d'influence »). Ce faisant, je poursuis mon exploration de la théorie de la dominance économique (TDE), mon dada depuis quelque temps déjà (cf. [LEB 17] pour une synthèse). Dans un graphe de la TDE, on peut simuler la diffusion de chocs exogènes au sein de la structure d'interactions. Ici, on pose un certain nombre de contraintes de sorte que la diffusion identifie un arbre orienté reliant tous les nœuds du graphe pour chaque source / nœud possible de choc. On ordonne ces arbres par leur valeur. On montre que les quelques arbres aux valeurs les plus élevées résument bien la structure des dominances du réseau, alors qu'ils ne contribuent que de manière infinitésimale à la valeur des dominances globales dans celui-ci. Des données benchmark sont utilisées pour mettre en évidence ce résultat de la TDE (commerce international). "Il n'y a plus qu'à » exploiter des matrices de flux technologiques avec cette technique, matrices qui posent cependant aujourd'hui encore quelques problèmes opérationnels pour une application immédiate, liés à leur taille (nombre de nœuds).

François-Xavier Meunier revisite les modèles de diffusion technologique en étendant leur base structurale (« La diffusion des technologies d'ingénierie biomédicale : évidences sur données des brevets »). C'est un exercice qui a déjà été entrepris dans le passé récent, mais de manière incomplète. La diffusion, ici, ne concerne pas l'usage, mais la production des technologies. Avec quelles autres technologies se combinent-elles pour produire de l'invention ? L'outil de base est le graphe de cooccurrences, mais seule l'information sur le nombre des technologies connectées à celle évaluée est utile. François-Xavier montre alors qu'il y a deux manières d'envisager la question. La première consiste à se placer à une date précise et à compter les technologies connectées à celle dont on cherche à étudier la diffusion. Il y a diffusion si, dans le temps historique, ce nombre augmente, indépendamment de l'identité des technologies connectées. Pour François-Xavier, cette première approche pose problème dans le sens où les effets de la cumulativité dans la création des connaissances sont effacés : ce qu'on savait faire hier ne compte pas pour expliquer la situation actuelle. Il propose donc un cadre qui intègre la cumulativité dans la production de connaissances technologiques, et il (C) 2021 ISTE OpenScience - Published by ISTE Ltd. London, UK - openscience.fr 
parvient ce faisant à simuler la diffusion des technologies en matchant ses données sur longue période avec des courbes logistiques. Ce qui permet d'identifier les technologies possédant, à court ou à plus long terme, les potentiels de diffusion / d'ubiquité les plus élevés.

Dernier article du numéro : Richard Le Goff aborde le thème de la convergence technologique ( $«$ La convergence technologique : le cas du véhicule autonome électrique »). Pour lui, cette convergence peut en partie expliquer pourquoi aujourd'hui des industries autrefois organisées en silo s'entremêlent. Le cas du véhicule autonome électrique est particulièrement représentatif de ces dynamiques industrielles contemporaines où des marchés nouveaux apparaissent (« marchés augmentés »). L'émergence de nouvelles industries résulte de la convergence d'anciennes. Comme il le note justement, cette forme d'émergence reste un angle mort de l'économie industrielle. Depuis les travaux de Steven Klepper, on sait modéliser le cycle de vie d'une industrie créée ex nihilo. Avec les travaux sur les systèmes technologiques d'innovation, on peut également envisager les situations où la technologie favorise des mouvements de parcellisation / éclatement ou, au contraire, de concentration des bases industrielles, mais sans que l'on puisse modéliser l'évolution à long terme de ces mouvements. Richard propose une approche qualifiée de « téléologique » où l'émergence industrielle est posée, et où il s'agit de repérer les conditions technologiques de cette émergence. Pour cela, il utilise la théorie de la dominance économique et propose quelques indicateurs simples pour valider l'hypothèse de la convergence technologique entre les domaines de l'énergie, des transports et des télécommunications. Il montre empiriquement, sur longue période, que ces domaines sont de plus en plus présents ensemble dans des mêmes inventions, rendant " l'émergence industrielle par la convergence technologique » envisageable.

Que de chemins parcourus depuis dix ans ! L'analyse des réseaux sociaux, la théorie de la dominance économique et la «nouvelle science des réseaux » sont amenés à être mobilisées de manière croissante en économie de l'innovation. Pas de surprise en cela, me semble-t-il. Lorsque Adam Jaffe et ses collègues produisent des matrices de flux territoriaux de connaissances technologiques, ils prêtent leurs études à une interprétation en termes de graphes. Même chose pour Frederic Scherer et les matrices de flux technologiques. Comme ce fut le cas dans les années 1970 avec les matrices d'échanges interindustriels étendues par la théorie mathématique des graphes. En économie de l'innovation, cette « augmentation », pour reprendre la terminologie de Richard Le Goff, est encore à poursuivre. Ce numéro de Technologie et Innovation propose un état des lieux en 2021. Rendez-vous dans quelques années pour un autre point d'avancement.

\section{Bibliographie}

[ELO 16] ElOUAER-MriZAK S., PICARD F., « Dynamique technologique et politique régionale d'innovation : l'apport de l'analyse statistique des réseaux », Innovations, $n^{\circ}$ 50, p. 13-41, 2016.

[FRI 19] FRIgANT V., Miollan S., PRESSE M., VIRAPIN D., «Quelles frontières géographiques pour les systèmes d'innovation technologique ? Une analyse par les co-brevets appliquée au véhicule à pile à combustible », Innovations, $\mathrm{n}^{\circ} 58$, p. 243-273, 2019.

[KRA 11] KRAFFT J., QUATRARO F., SAVIOTTI P.P., « The knowledge-base evolution in biotechnology: a social network analysis », Economics of Innovation and New Technology, vol. 20, p. 445-475, 2011.

[LEB 17] LeBert D., El Younsi H., Dynamiques des spécialisations internationales, ISTE Press, Londres, 2017.

[WAS 94] WASSERMAN S., FAUST K., Social network analysis: methods and applications, Cambridge University Press, Cambridge (Mass), 1994. 